\title{
Hacia una definición conversacional de la manipulación*
}

\author{
Pablo Porto López ${ }^{* *}$ \\ Cristián Santibáñez ${ }^{* * *}$
}

\section{Resumen}

Este trabajo aborda la manipulación desde una perspectiva conversacional capaz de identificar los mecanismos subyacentes a un intercambio calificado socialmente como "manipulativo". En toda manipulación tiene lugar un desplazamiento entre dos cuestiones diferentes: una cuestión $\left(\mathrm{Q}_{2}\right)$, no explicitada en el intercambio comunicacional, es presentada por el agente manipulador como otra cuestión $\left(Q_{1}\right)$ que, sin embargo, es capaz de convocar una respuesta $\left(\mathrm{R}_{1}\right)$ del destinatario, que estará conectada de manera significativa con la respuesta que suscitaría la primera cuestión $\left(R_{2}\right)$. La relación entre ambas respuestas podrá ser de: equivalencia, inclusión/intersección o consecución. Algunas de las ventajas de esta definición de manipulación son que así se evita colocar como rasgos definitorios de la manipulación, entre otros, a la intencionalidad del agente manipulador y al perjuicio sufrido por el sujeto manipulado, a la mentira de uno y al error interpretativo del otro, rasgos no necesarios ni suficientes para decir que ha tenido lugar un acto de manipulación.

Palabras clave: Desplazamiento, dinámica conversacional, proceso manipulativo, toma de turnos dialógicos.

\section{Towards a conversational definition of manipulation}

\begin{abstract}
Manipulation appears to analysis as an essentially multiform and heteroclite object. This work approaches manipulation from a conversational perspective that is capable of identifying the mechanisms that underlie an exchange that is socially qualified as "manipulative". We postulate here that in any manipulation there is a displacement between two different questions: a question $\left(\mathrm{Q}_{2}\right)$, which does not appear explicitly in the communicational exchange, is presented by the manipulating agent in terms of another question $\left(Q_{1}\right)$ which then triggers a response $\left(\mathrm{R}_{1}\right)$ from the recipient that will be connected in a meaningful way with the answer the first question raises $\left(\mathrm{R}_{2}\right)$. The relationship between both responses, which the manipulated agent should not be able to establish if the strategy wants to be successful,
\end{abstract}

Este artículo forma parte de una línea de investigación en torno a la comunicación, la argumentación y el análisis del discurso que los autores han desarrollado desde hace más de una década.

Argentino. Magíster en Análisis del Discurso y doctorando en Lingüística de la Universidad de Buenos Aires. Académico de la Universidad de Buenos Aires, Buenos Aires, Argentina. pportolopez@ sociales.uba.ar

*** Chileno. Doctor en Lingüística de la Universidad de Houston. Académico de la Universidad Católica de la Santísima de Concepción, Concepción, Chile. csantibanez@ucsc.cl 
could be at least of three types: equivalence, inclusion/intersection or consecution. Some of the advantages that result from a definition of manipulation in terms of a conversational exchange, are that it avoids placing as defining features of the manipulation, among others, the intentionality of the manipulating agent and the damage suffered by the manipulated subject, the lie of one and the interpretative error of the other, traits that are neither necessary nor sufficient for it to be said that an act of manipulation has taken place.

Keywords: Displacement, Conversational dynamic, Manipulative process, Dialogue turn-taking.

\section{Introducción}

Hoy la perspectiva cognitiva ejerce un reinado casi indiscutible cuando hablamos de fenómenos lingüísticos (Fillmore, 1982; Lakoff, 1993, 1994; Lakoff \& Johnson, 1980; Langacker,1987). En la literatura contemporánea respecto del problema de la manipulación —como se verá luego-, tiende a pasar lo mismo. En este trabajo no pretendemos derrocar este reinado, sino ofrecer ideas complementarias a varias ya existentes que intentan dar una definición de manipulación, incorporando una perspectiva dialógica y/o conversacional, vale decir, interactiva.

Nuestro objetivo es articular las dimensiones de la acción y la de los estados mentales con la dimensión conversacional, sosteniendo que existe algo que puede ser llamado interacción conversacional manipulativa, esto es, que la manipulación tiene rasgos producidos durante la interacción que le son propios, lo que supone tratar con la objeción según la cual la manipulación o, más precisamente, la intención manipulativa, no tiene estatus comunicativo esencial en la interacción entre enunciador (manipulador) y destinatario (manipulado).

Desde el punto de vista léxico, una primera dificultad para delimitar la noción de manipulación se refiere a distinguirla del cercano concepto de influencia. Para las definiciones del lenguaje ordinario, manipular consiste en "manejar o influenciar hábilmente, especialmente de manera injusta" (Webster's Encyclopedic, 1996). Si se asimila manipulación a influencia, se puede pensar la escena manipulativa sirviéndose de las observaciones de Bremond (1970) acerca del rol del influenciador. Bremond afirma que la influencia consiste en la interacción de dos agentes, uno como influenciador y el otro como paciente influenciado, de quien el primero modifica las disposiciones respecto de "una situación 
presente, o de acontecimientos futuros en los cuales es susceptible de participar, sea solo como paciente, sea como paciente y agente a la vez" (Bremond, 1970, p. 60). Una pregunta posible a esta forma de ver el problema es la siguiente: ¿es solamente ese rasgo axiológicamente negativo — su condición de acto éticamente reprobable o injusto-, lo que distingue a la manipulación de la noción más amplia de influencia? Según van Dijk (2006) la manipulación se distingue por ser una forma de interacción ilegítima, pero además

en la persuasión los interlocutores son libres de creer o actuar como les plazca, según si aceptan o no los argumentos de quien persuade, mientras que en la manipulación a los receptores se les asigna, típicamente, un papel más pasivo: son víctimas de la manipulación (p. 51).

Procuraremos profundizar más adelante en las razones por las que suele atribuírsele un rol más pasivo al sujeto manipulado.

Otra dificultad que presenta el fenómeno para las definiciones de uso corriente tiene que ver con la diversidad de criterios convocados. Así, manipular consistiría en "intervenir con medios hábiles y, a veces, arteros, en la política, en el mercado, en la información, etc., con distorsión de la verdad o la justicia, y al servicio de intereses particulares" (Real Academia Española, RAE, 2014). Se hace referencia en esta definición a cuestiones tan disímiles como el interés particular de quien manipula, la distorsión de la verdad o de la justicia (los medios arteros), o la intervención en determinadas esferas de la vida social (política, económica y de la información); sin embargo, no se trata de otra cosa que de la descripción de una estrategia de medios, esto es, el empleo de medios hábiles y arteros para la satisfacción de un interés particular.

Esta segunda definición parecería indicar que un abordaje del fenómeno de la manipulación que se pretenda completo requerirá de una teoría de la acción. En tanto la manipulación se consuma en la práctica y se observa por sus efectos, exigiría la consideración de los comportamientos a los cuales induce y las consecuencias que trae aparejadas. Pero al mismo tiempo, la definición de la RAE da cuenta del modo en que la manipulación se vincula con las intenciones y las estrategias del manipulador. Landowski (2005) sostiene que

el 'manipulador' (o estratega) en potencia, atribuye a su socio (...) un estatuto semiótico idéntico al que reconoce para sí 
mismo: el de un sujeto (...) Toda elección estratégica expresa esencialmente la manera en que el manipulador construye la competencia (cognitiva, epistémica, volitiva, deóntica, etc.) del otro, y en que localiza los puntos sensibles, las fallas o las zonas críticas susceptibles, a sus ojos, de volver a su interlocutor, precisamente, manipulable (...) evidentemente, a riesgo de equivocarse de cabo a rabo (p. 147-150).

Si se tiene esto en consideración, el análisis de la manipulación requeriría asimismo de una teoría de la mente, o de los estados mentales, que abarque el cálculo del manipulador (básicamente la intención), y también el modo en que el destinatario (manipulado) procesa la información que se le provee.

Para dar cuenta de estos desafíos conceptuales, en la segunda sección discutimos parte de la reflexión proveniente de la teoría de los actos de habla que puede estar conectada con el fenómeno manipulativo como tipo de acto de habla; en la tercera sección ofrecemos nuestra perspectiva, incluyendo una propuesta específica referida a entender el proceso manipulativo como un desplazamiento entre cuestiones distintas, pero conectadas, que promueve el agente manipulador para lograr un efecto práctico, ofreciendo en este apartado los tipos de relaciones que pudieran darse entre estas cuestiones distintas; en la cuarta sección analizamos un caso empírico en el que se puede observar un proceso manipulativo, que extraemos de la realidad política internacional reciente; para concluir con la quinta sección, que corresponde a las conclusiones, enfatizamos los puntos centrales de nuestro ángulo, resaltando que el fenómeno manipulativo jamás tiene un carácter convencional.

\section{Acción, intención y lenguaje: lo que la manipulación - no- hace lingüísticamente}

En Actos de habla (2001), Searle afirma que hablar una lengua consiste en participar de un tipo de conducta intencional gobernada por reglas. Pero precisa luego que estas intenciones son de un tipo particular, específica a los actos de habla: "solamente ciertos géneros de intenciones son adecuados para la conducta que denomino actos de habla" (p. 26). Según el principio de expresabilidad, cualquier cosa que se tenga la intención de decirse puede — al menos potencialmente-, 
ser dicha: "para todo posible acto de habla, existe un posible elemento lingüístico cuyo significado (dado el contexto de emisión) es suficiente para determinar que su emisión literal constituye una realización de, precisamente, ese acto de habla" (p. 30).

Así, la noción de intención en Searle está vinculada estrechamente con la de significado: la emisión de una oración es un medio convencional de intentar producir un efecto en el oyente, pero no un efecto práctico, sino un efecto ilocucionario. La crítica de Searle (2001) a Grice (2011) consiste precisamente en que este último concibe el significado en términos de intentar realizar un acto perlocucionario, esto es, provocar algún comportamiento o respuesta en el oyente. Para Searle, la intención del hablante nada tiene que ver con esto. Una noción de significado - y en esto radica justamente su crítica a Grice-, debe mostrar la conexión entre el hecho de que un hablante quiera decir algo mediante lo que enuncia y lo que efectivamente significa aquello que dice de acuerdo con el lenguaje. La intención de un hablante que realiza un acto de habla se limita a producir un efecto ilocucionario en el oyente, intentando que este reconozca su intención de producir ese efecto, y lo hace sirviéndose de las reglas que gobiernan el uso de las expresiones empleadas que las asocian a la producción de dicho efecto. En palabras de Searle (2001):

Quiero argumentar que decir algo queriendo decir lo que significa consiste en intentar realizar un acto ilocucionario (...) En el caso de los actos ilocucionarios logramos hacer lo que intentamos hacer, al conseguir que nuestro auditorio reconozca lo que estamos intentando hacer. Pero el 'efecto' sobre el oyente no es ni una creencia ni una respuesta, consiste simplemente en la comprensión, por parte del oyente, de la emisión del hablante. Es este efecto lo que he estado denominando efecto ilocucionario (p. 54-56).

Queda claro, entonces, que la intención manipulativa no puede ser de este género. La manipulativa ha sido descrita como una intención que, todo lo contrario a lo que el hablante pretende que ocurra en un acto ilocucionario, no puede ser reconocida por el oyente si se espera que la estrategia de manipulación resulte exitosa. Desde luego, en un diálogo en el que hay una manipulación encontramos la intención de la que habla Searle, la que hace posible que el hablante pueda producir el efecto ilocucionario, pues para que haya manipulación 
el oyente tiene que comprender lo que el hablante le dice, es decir, tiene que haber algún tipo de intercambio significativo. Sin embargo, esta constatación poco dice acerca de los rasgos que hacen que dicha emisión pueda calificarse como un discurso manipulativo. Es decir, y dicho de la forma más directa posible, las condiciones de felicidad de la emisión prototípica de un acto de habla - a saber, que la intención ilocucianaria sea reconocida-, no servirían para un acto de habla que procura la manipulación del oyente, ya que esta última funciona sobre el no reconocimiento. No hay relación convencional entre intención y significado en la manipulación.

Es así que la manipulación no puede considerarse un acto de habla, puesto que no es posible emplear un indicador explícito de fuerza ilocucionaria. Tal como lo señalan Maillat y Oswald (2009), el acto manipulativo no puede traducirse a un performativo con la forma $F(p)$ : "Yo te manipulo + contenido proposicional" (p. 352). Pero, especialmente, la manipulación no puede ser un acto de habla, puesto que no juega ningún papel el principio de expresabilidad de Searle: la relación entre el acto manipulativo llevado a cabo mediante $E$ (la expresión lingüística) y el significado de $E$ no es jamás directa (considerando incluso el contexto apropiado), como sí lo es en un acto de habla asertivo, por ejemplo.

Entonces, si la manipulación no se rige por los criterios de los actos ilocucionarios, tal vez podamos encontrarlos por el lado de sus efectos, esto es, los actos perlocucionarios. Al respecto, Searle (2001), que retoma en este punto a Austin (1998), los define como "las consecuencias o efectos que tales actos (los ilocucionarios) tienen sobre las acciones, pensamientos o creencias, etc., de los oyentes" (p. 34). Este tipo de actos, a diferencia de los ilocucionarios, no dependen de las reglas constitutivas del lenguaje, en el sentido en que no son convencionales. Por eso los efectos perlocucionarios perseguidos por los turnos conversacionales pueden permanecer ocultos.

El acto perlocucionario se resuelve en la realidad, en los hechos brutos y, en general, no aparece como determinado por el acto de habla, pues interviene en esta instancia toda una serie de variables que no tiene que ver necesariamente con el lenguaje. Se dice que en general el acto perlocutivo no aparece determinado por el tipo de acto de habla; una excepción posible podría ser el caso del acto de prometer que, 
sinceramente emitido, genera - por el tipo de acto de habla que es-, la expectativa material que contiene ${ }^{1}$.

Ahora bien, si la manipulación parecería circunscribirse al ámbito de este tipo de efectos en la interacción dialógica de carácter no convencional es necesario, no obstante, efectuar ciertas precisiones respecto de la posibilidad de asimilar la manipulación a los efectos perlocucionarios de los actos de habla.

Como el mismo Searle señala, el hablante puede, mediante una aseveración que provee información, convencer al oyente, siendo el convencer un efecto perlocucionario del acto ilocucionario de aseverar. Del mismo modo, una petición o una amenaza (acto ilocucionario) pueden lograr que el oyente haga algo (acto perlocucionario). Aun tomando en consideración el hecho de que una aseveración puede perfectamente no convencer, y que un pedido puede no lograr que alguien haga aquello que se le pide, resulta evidente que la relación que se establece entre el acto ilocucionario y el perlocucionario es, si bien no convencional, al menos socialmente previsible; y lo que es más importante aún aquí: lo es para el destinatario en el contexto de la situación de comunicación. En el horizonte de expectativas de un pedido se encuentra la acción de aquel a quien se le pide que haga algo (tanto en el horizonte del hablante como en el del oyente). Este tipo de conexión entre acto ilocucionario y acto perlocucionario, esta previsibilidad, no existe en el caso de la manipulación, al menos en el sentido de una previsibilidad social (y del destinatario) como opuesta a una previsibilidad estratégica (vinculada a un cálculo) y contextualizada, por parte del agente manipulador.

La manipulación como recurso atiende a un móvil (meta, objetivo, interés) del agente manipulador. Cuando se origina por un móvil pragmático, la acción es concebida por el agente como el medio a emplear para la realización de otra tarea: su elección responde a un interés o cálculo (Bremond, 1970, p. 64). En este sentido, puede resultar esclarecedora la comparación entre dos actividades tan distintas como prometer y pescar, que el propio Searle (2001) delinea, en tanto que la manipulación (¿extrañamente?) parecería colocarse más cerca de la segunda que de la primera:

Precisamente, Searle (2002) vuelve sobre este tipo de acto de habla para esclarecer lo que lo distingue de otros. 
[E]n el caso de pescar, las relaciones fines-medios, esto es las relaciones que me facilitan o me capacitan para alcanzar mi meta, están constituidas por hechos físicos naturales, hechos tales como, por ejemplo, que el pez muerda el anzuelo (...) existen, de hecho, técnicas, procedimientos e incluso estrategias que los pescadores afortunados siguen, y sin duda todos esos procedimientos incluyen en algún sentido reglas (regulativas). Pero que bajo tales y cuales condiciones una persona atrape un pez no es un asunto de convención ni de nada parecido a una convención. Por otro lado, en el caso de los actos de habla realizados dentro de un lenguaje, es un asunto de convención - en tanto que opuesto a estrategia, técnica y procedimiento o hecho natural - el que la emisión de tales y cuales expresiones cuente bajo ciertas condiciones como el hacer una promesa (p. 46-47).

De este modo, la intención en la manipulación podría concebirse como una acción racional, es decir, como una disposición de medios adecuados para la consecución de un propósito específico. Es lo que también se denomina razonamiento práctico, y que puede expresarse como esquema argumentativo (Macagno, 2014, p. 112):

Premisa 1: Un agente posee un objetivo $\mathrm{O}$.

Premisa 2: La acción A es un medio para alcanzar O (a menos que A se lleve a cabo, O no será alcanzado).

Conclusión: Por lo tanto, A debe ser llevado a cabo.

Algunas de las preguntas críticas de este esquema, y que tienen que ver con el cálculo del agente, son: ¿Qué acciones alternativas a A, que producirían asimismo el objetivo $\mathrm{O}$, deberían ser consideradas?, o ¿cuál es (A o alguna otra) la acción más eficiente para alcanzar O? (Macagno, 2014, p. 112).

En definitiva, se puede concluir hasta el momento que la teoría de los actos de habla no ofrece herramientas adecuadas para dar cuenta del modo en el que opera la manipulación, puesto que, como vimos, la intención ilocucionaria hace referencia a una dimensión convencional de la comunicación (relativa a las reglas constitutivas del lenguaje) que el hablante espera que el oyente reconozca. Asimismo, decir que la manipulación se encuentra del lado de los efectos perlocucionarios, 
tampoco arroja luz sobre el fenómeno en tanto no contribuye al análisis de la estrategia del agente manipulador y del funcionamiento propiamente manipulativo en el marco de un diálogo o conversación.

Queda claro que cuando se habla de intención manipulativa, se está empleando la noción de intención en un sentido que nada tiene que ver con el de intención ilocucionaria en Searle. Por ello, sería un error siquiera suponer que la fuerza ilocucionaria de la intención manipulativa debería poder expresarse en la forma de un performativo explícito. Ahora bien, ¿el hecho de que la manipulación no sea un acto de habla significa que no puede ser analizada como fenómeno conversacional?

\section{La manipulación como fenómeno conversacional}

El carácter heterogéneo de la manipulación constituye, según Maillat y Oswald (2009), la razón fundamental por la cual no se ha llegado a ningún consenso para la definición de la misma en el seno de las humanidades. Así, puede observarse por ejemplo que la definición elaborada por van Dijk (2006) incluye la consideración de aspectos sociales, cognitivos y discursivos:

la manipulación es un fenómeno social, especialmente debido a que involucra la interacción y el abuso de poder entre grupos y actores sociales, un fenómeno cognitivo debido a que la manipulación siempre implica la manipulación de las mentes de los participantes y un fenómeno discursivo-semiótico, porque la manipulación se ejerce mediante la palabra oral o escrita y los mensajes visuales (p. 52).

Los diversos intentos por comprender el fenómeno no serían para Maillat y Oswald (2009) más que intentos parciales y descriptivamente inadecuados, pues no harían más que elaborar una lista de características del fenómeno manipulativo, ninguna de las cuales para estos autores-, constituye una condición necesaria ni suficiente para la manipulación. Los criterios identificados y examinados por los autores son cinco: las condiciones de verdad y de felicidad del enunciado manipulativo; el interés del hablante; el carácter encubierto (covertness) de la manipulación; la desigualdad social entre manipulador y manipulado; y, por último, la intención de engañar (deceptive intention). 
No todos los criterios analizados por estos autores poseen para nosotros la misma importancia: solamente tres de ellos deben ser considerados seriamente. Maillat y Oswald (2009) demuestran convincentemente que la manipulación no depende de que aquello que se diga sea verdad o no, ni tampoco de que emerja necesariamente de relaciones sociales asimétricas. Se puede manipular diciendo la verdad, como también puede perfectamente darse el caso de que un amigo manipule a otro, que haya manipulación en una pareja, esto es, sin que haya a priori relaciones de poder.

El criterio del interés del hablante, y su consecuencia atribuida de perjuicio sobre el oyente constituye un aspecto más delicado, pues en el término manipulación existe, como se ha mencionado, una fuerte carga axiológica. En un caso en el que se manipule al oyente para el beneficio de este y sin que esto comporte rédito alguno para el hablante, difícilmente se hablaría de manipulación. En este sentido, se debería recurrir a un adjetivo calificador, como en la expresión "manipulación altruista" (Santibáñez, 2017), lo que remarca el carácter negativo del sentido original del término. Así, el interés del hablante no parece un criterio que haya que tener en cuenta para la definición de la manipulación, si bien casi siempre se satisface cuando se analizan procesos manipulativos concretos.

Esto nos deja con dos criterios a considerar: el carácter oculto de la manipulación y la intención de manipular. Consideraremos este último y dejaremos el estudio del carácter oculto de la misma para más adelante. La propuesta de Maillat y Oswald consiste en considerar que la manipulación no es identificable a partir de ninguna de estas cualidades (solas o combinadas), sino que se define por los límites que impone a la capacidad del destinatario para representarse la información crítica relativa al enunciado en cuestión. Los autores sostienen que, en el estudio de la manipulación, el foco debe estar no en la intención manipuladora del hablante (la cual no tiene ningún status en la interacción comunicativa), sino en el proceso cognitivo de interpretación del oyente. La manipulación consiste, entonces, en "un conjunto de restricciones que limita los procesos de selección contextual del destinatario" (Maillat \& Oswald, 2009, p. 361).

Sin embargo, nuestra observación a esta propuesta sería que el problema de focalizar la atención por completo en el destinatario reside en que la manipulación ya no se distingue de otros procesos cognitivos 
en los que hay un error o un sesgo interpretativo. El proceso cognitivo por el que opera la manipulación es analizado por estos autores desde los postulados de la teoría de la relevancia (Sperber \& Wilson, 1995). Entre aquellos postulados destaca la idea de que solo algunos de los supuestos contextuales son repuestos por el destinatario en la interpretación del enunciado que le es ofrecido. De esta forma, la manipulación es descrita como un proceso en el que el destinatario interpreta el enunciado en un contexto reducido (C), sin tener acceso a los supuestos contextuales alternativos más amplios (C'), que podrían desenmascarar algún objetivo oculto.

Además, y esta es una segunda observación que le hacemos a la propuesta de Maillat y Oswald (2009), la intencionalidad del hablante se reintroduce inadvertidamente en la definición de manipulación que los autores ofrecen, en tanto que sin ella parecería perderse de vista la especificidad del fenómeno manipulativo. Así, estos autores afirman que "la manipulación es un intento de engañar al destinatario que asegura que este solamente acceda a un conjunto limitado de los supuestos contextuales, por medio de un aprovechamiento de su optimismo cognitivo" (p. 369). No solo se habla de intención, sino de intención de engañar o despistar (attempt at misleading). En este sentido, Maillat y Oswald montan la interacción manipulativa sobre el proceso cognitivo más amplio del error o el sesgo, estableciendo una continuidad entre ambos: "El giro comunicativo introducido por el discurso manipulativo tiene que ver con las restricciones externas impuestas por el manipulador al proceso interpretativo, el cual permanece inalterado" (p. 362). Abordada desde este punto de vista -y prescindiendo de la intención manipulativa-, el aspecto propio de la manipulación parecería perderse.

Entonces, ¿puede describirse el modo en que funciona la manipulación sin recurrir a la noción de intención del hablante? Del mismo modo que otros tipos de acciones que requieren una planificación estratégica, puede afirmarse que la manipulación es generalmente intencional. Sin embargo, es posible e incluso conveniente teóricamente, definirla en términos conversacionales sin apelar a su naturaleza intencional por dos razones. Por una parte, está el hecho, señalado por Maillat y Oswald, de los casos en que existe manipulación no intencional. Esta tiene lugar cuando el agente inmediato manipula sin saberlo, por ejemplo, cuando se repite un rumor manipulativo del que no ha sido el autor. Esta manipulación de segunda mano no parece 
ser manipulación en sentido estricto, pues nadie diría "lo manipulé sin saberlo"; simplemente diría: "fuimos los dos manipulados" o, llegado el caso, "fui un instrumento para su manipulación (la de un tercero)". De cualquier manera, es incontestable que los efectos manipulativos tienen lugar aunque no exista intención manipulativa del agente inmediato. Por ello, en este caso no puede considerarse a la intención como la causa material de la manipulación, aunque sí como su causa eficiente (la intención del autor del rumor).

Pero existe una segunda razón, más importante aún, para prescindir de la noción de intención a la hora de definir la manipulación. Definir un intercambio comunicativo en términos de la intención de producir un efecto perlocucionario o práctico determinado, resulta muy problemático en términos teóricos, pues supone reducir en última instancia las propiedades de dicho intercambio a las motivaciones internas (subjetivas) del hablante, por un lado, y a las consecuencias efectivas en términos de hechos brutos (no convencionales), por el otro.

Una manera posible de resolver este puzle teórico sería tomar como inspiración el modo en que Meyer (2013) y Plantin (1993) conceptualizan el fenómeno argumentativo. Para estos autores, la argumentación se estructura a partir de una pregunta o cuestión, que admite al menos dos respuestas diferentes. Nosotros podríamos decir, para el caso de la manipulación, que esta en cambio involucra necesariamente dos cuestiones diferentes. No se trataría meramente de un ocultamiento de información relativo a una cuestión, sino de un procedimiento por el cual se reemplaza una cuestión por otra, o se plantea una cuestión en términos de otra, lo que hace posible mantener oculto el objetivo ulterior del manipulador. Así, el mecanismo del discurso manipulativo consistiría en presentar una cuestión $\mathrm{Q}_{1}$, la cual supone una respuesta por parte del destinatario que, a los fines prácticos (o considerada en términos de efectos perlocucionarios), constituye (como mínimo) un paso en la dirección de la consecución de los objetivos concernientes a la cuestión no explicitada $\mathrm{Q}_{2}$. Esta forma de ver las cosas, como se observa, pone énfasis en el proceso interactivo y/o conversacional. En esta perspectiva, además, la manipulación no es convencional y no está relacionada directamente con el sentido, puesto que $\mathrm{Q}_{2}$ no está explicitada (ni el contenido de lo que sería una respuesta adecuada a $\mathrm{Q}_{2}$ ), ni tampoco una consideración detenida de $\mathrm{Q}_{1}$ permitiría al destinatario inferirlo. 
Maillat y Oswald (2009) parecen advertir que en la manipulación existen dos cuestiones diferentes cuando sostienen que "es importante distinguir entre, por un lado, la movida constrictiva e inherentemente manipulativa y el (frecuentemente) inocuo enunciado cuya interpretación está siendo limitada (constrained), por el otro lado" (p. 363). Sin embargo, profundizan solamente en los aspectos cognitivos del proceso interpretativo que lleva al error o al procesamiento superficial (shallow processing) por parte del destinatario. Además, como veremos más adelante, postulan el sesgo en términos de una única cuestión cuyos supuestos contextuales han sido constreñidos de alguna manera.

Ahora bien, ¿qué tipo de relaciones se establecen en la manipulación entre las dos cuestiones $\mathrm{Q}_{1}$ y $\mathrm{Q}_{2}$ ? Es evidente que cualquier reemplazo de una cuestión por otra no producirá una manipulación. Más allá del simple y llano error, una "estrategia evasiva" en la que se plantea $\mathrm{Q}_{1}$ (por ejemplo, un comentario de un marido a su mujer acerca de lo atractiva que se ve) simplemente para distraer su atención respecto de $\mathrm{Q}_{2}$ (el hecho de que ha llegado tarde a una cita), no desencadenará necesariamente una situación de manipulación.

Entonces, sostendremos que para que haya manipulación, $\mathrm{Q}_{1}$ y $\mathrm{Q}_{2}$ deberán estar vinculadas de una manera significativa, no convencional $\left(Q_{1}\right.$ no significa, no implica y no plantea de manera subyacente $\left.Q_{2}\right)$, sino práctica. Las posibilidades lógicas que aparecen en tanto que tipos ideales son al menos tres: la relación entre la cuestión $\mathrm{Q}_{1}$ explicitada o aludida por el enunciado $E$ y que convoca una respuesta $\mathrm{R}_{1}$ por parte del destinatario, y la cuestión $\mathrm{Q}_{2}$ que suscita una respuesta $\mathrm{R}_{2}$, cuestión que no se plantea, pero cuya respuesta constituye el objetivo ulterior del agente manipulador, puede ser de equivalencia, de inclusión/ intersección o de consecución.

- Equivalencia: $\mathrm{R}_{1}$ es hacer $\mathrm{R}_{2}\left(\mathrm{R}_{1}=\mathrm{R}_{2}\right)$.

- Inclusión/intersección: $\mathrm{R}_{1}$ es hacer parcialmente $\mathrm{R}_{2}\left(\mathrm{R}_{1} \subset \mathrm{R}_{2} \mathrm{o} \mathrm{R}_{1} \cap \mathrm{R}_{2}\right)$.

- Consecución: $R_{1}$ no es equivalente $\mathrm{R}_{2}$ ni constituye una parte de realizar $\mathrm{R}_{2}$, sino que $\mathrm{R}_{1}$ es un paso previo o preparatorio en la dirección de conseguir $\mathrm{R}_{2}\left(\mathrm{R}_{1 \ldots} \mathrm{R}_{2}\right)$.

La primera categoría, la equivalencia $\left(R_{1}=R_{2}\right)$, parece la más difícil de concebir en un proceso manipulativo, si se supone que el objetivo ulterior del agente manipulador debe en todos los casos no aparecer como evidente al destinatario. Imaginemos un caso en el que el dueño de una 
empresa se propone incorporar a un nuevo gerente. Luego del proceso de selección han quedado solo dos candidatos para el puesto. Un segundo empresario, conocido del primero, le sugiere contratar al candidato A - para lo que puede referirse a la buena reputación que tiene A en el ambiente de los negocios-, con el objetivo de que B, que es empleado actualmente de su firma (hecho desconocido por el primer empresario) no abandone su actual trabajo (pues la oferta del primer empresario es mejor económica y profesionalmente). La finalidad no declarada del segundo empresario es evitar tener que desprenderse de los servicios de $B$, de quien sabe es un mejor empleado que A, lo que supondría un perjuicio para el primer empresario. Sin embargo, no es necesario que los argumentos en favor de contratar a A sean falsos: puede perfectamente darse que A sea un buen candidato, solo que B es uno mejor. El acto de habla es una aseveración y las condiciones de sinceridad se satisfarían, en principio. Quizás, si asumiéramos las máximas conversacionales de Grice (2011), se podría desnudar la dimensión manipulativa del enunciado del segundo empresario, ya que su comunicación violaría la máxima de cantidad: haz tu contribución tan informativa como se requiera para los propósitos del intercambio informativo. En efecto, operaría algún tipo o grado, de insinceridad.

Desde este último punto de vista, el manipulador —aquí el segundo empresario-, presenta de manera explícita la cuestión $\mathrm{Q}_{1}\left(i_{\text {Conviene }}\right.$ más al primer empresario contratar a $\mathrm{A}$ o a B? o ¿Quién se desempeñaría mejor como gerente: $\mathrm{A}$ o $\mathrm{B}$ ?) mientras que $\mathrm{Q}_{2}$ (¿Cómo evitar perder los servicios de $\mathrm{B}$ ?), cuestión que en realidad está en juego para el segundo empresario, no se expresa en ningún momento (ni puede ser restituida por el destinatario, el primer empresario, pues no sabe que B trabaja para su interlocutor). Como puede verse, hacer $\mathrm{R}_{1}$ (hacer que el primer empresario contrate a A) es, en este caso, hacer $\mathrm{R}_{2}$ (conservar, por parte del segundo empresario, los servicios de B). Ciertamente este parece ser un caso límite, pues el objetivo ulterior del manipulador no debe ser identificado por el manipulado, y la identidad entre $R_{1} y R_{2}$ hace que no siempre sea posible mantener esto oculto.

Pasemos ahora a la segunda categoría: inclusión/intersección $\left(R_{1} \subset\right.$ $\mathrm{R}_{2} \mathrm{o} \mathrm{R}_{1} \cap \mathrm{R}_{2}$ ). Supongamos, nuevamente considerando a los empresarios de nuestro anterior ejemplo, que el segundo empresario se propone conseguir para sus parientes un trabajo en la empresa del primero (por el motivo que sea: la empresa es un buen lugar para trabajar, los 
sueldos son altos, etc.). Imaginemos ahora que el candidato A, de quien el segundo empresario habla en términos laudatorios, es pariente suyo. Nuevamente, la cuestión que se plantea es $\mathrm{Q}_{1}\left({ }_{i}\right.$ Conviene más al primer empresario contratar a $\mathrm{A}$ o a $\mathrm{B}$ ?) mientras que $\mathrm{Q}_{2}$, que ahora tiene que ver con conseguir empleo a los parientes del segundo empresario, no aparece explicitada en ningún momento. El caso es diferente respecto del primer ejemplo, pues el hecho de que ingrese A supone una realización parcial del objetivo del segundo empresario, y no su concreción total (quedan aún otros parientes que incorporar a dicha empresa). Aquí la comunicación manipulativa, en tanto interacción en progreso, tendría que continuar para lograr su objetivo final.

En relación con la tercera categoría consecución $\left(R_{1 \ldots} R_{2}\right)$, y siguiendo con el ejemplo utilizado, el caso se observa cuando se asume que A es una persona de confianza del segundo empresario, y que el objetivo ulterior de este fuera que ingresara a la empresa para así obtener información corporativa de su competencia (pues planea expandir su compañía hacia el mismo rubro de producción que la empresa del primer empresario). El objetivo aquí consiste en hacerse de información corporativa secreta, para lo que es necesario que previamente A se ponga en la posición de tener acceso a ella. De este modo, $\mathrm{R}_{1}$ es un paso preparatorio en la dirección de alcanzar $\mathrm{R}_{2}$, pero no constituye su realización, y ni siquiera una realización parcial de dicho objetivo. La diferencia entre inclusión/intersección, por un lado, y consecución por el otro, no siempre es fácil de establecer. Se puede afirmar que en el caso de la consecución, si el objetivo ulterior no se alcanza, se podrá decir que no ha acontecido en absoluto el proceso manipulativo: por ejemplo, puede darse que la persona de confianza ingrese a la empresa, pero nunca tenga acceso a la información privilegiada que el segundo empresario deseaba conseguir. Mientras que en la inclusión/intersección, aunque no se concrete el objetivo ulterior, se dirá que ha sido parcialmente alcanzado: por ejemplo, de los cuatro parientes que se había propuesto colocar en la empresa, dos lo consiguieron. Con la tercera categoría, consecución, se deja ver también que ciertos procesos manipulativos pueden estar desfasados en el tiempo, vale decir, que los objetivos finales podrían alcanzarse entre distancias temporales importantes y que, incluso, pueden ser alcanzados por agentes que no fueron parte de las conversaciones y toma de turnos iniciales.

Esta tipología preliminar de relaciones entre $Q_{1} y_{2} Q_{2}$ en la manipulación, pretende restituir la idea general de objetivo manipulativo 
o estrategia de manipulación desde un punto de vista conversacional, atendiendo exclusivamente a las propiedades del enunciado (en contexto) sin tener que recurrir a la noción de intención. Y tampoco necesariamente a la idea de interés del hablante (y su contrapartida, el perjuicio del oyente), pues solamente se considera la conexión entre cuestiones de alguno de los tres tipos mencionados como mecanismo de base de la manipulación, lo que deja abierta la posibilidad a la manipulación altruista, además de la manipulación no intencional o de segunda mano.

Podemos ahora discutir el criterio para el reconocimiento de la manipulación que tiene que ver con el carácter oculto de la misma (covertness). Luego del recorrido realizado, resulta claro que el carácter oculto de la manipulación reside en que no exista evidencia explícita (ni circunstancial contextual) de la conexión entre $\mathrm{Q}_{1}$ y $\mathrm{Q}_{2} \mathrm{o}$, al menos, que el destinatario particular no sea capaz de realizar dicha conexión. Se puede decir, con Maillat y Oswald (2009), que la eficacia de la manipulación dependerá de que el destinatario no cuente con acceso a la información crítica, pero ahora podemos observar de manera precisa el modo y el lugar en el que dicha restricción opera: en el vínculo entre $\mathrm{Q}_{1}$ y $\mathrm{Q}_{2}$; vínculo que el locutor crea por medio de un cálculo estratégico y que, para que la manipulación sea exitosa, el destinatario no debe ser capaz de reponer. Así, razonamiento práctico (del locutor) y sesgo interpretativo (del oyente) son las caras, a uno y otro lado, del intercambio conversacional, del mismo proceso manipulativo.

\section{4. ¿Mentir es lo mismo que manipular? Análisis de un caso empírico}

El ejemplo aparece en Maillat y Oswald (2009), quienes aplican los principios de la teoría de la relevancia para su análisis. Los autores relatan el modo en que el gobierno de George W. Bush supuestamente manipuló a la opinión pública norteamericana para que interpretara la relevancia de la guerra contra Irak en el contexto de los ataques del 11 de septiembre. Según los autores, el gobierno de los Estados Unidos necesitaba construir un caso para atacar a Irak, y para ello Bush sostuvo en su State of the Union Address que "el gobierno británico se ha enterado que Saddam Hussein buscó recientemente cantidades significativas de uranio de África" (p. 367). Dicha información que la inteligencia americana había 
recabado y sabía que era falsa (o al menos dudosa), fue de todos modos utilizada con el fin de convocar una serie de supuestos contextuales para interpretar el enunciado siguiente: "Debemos (EE.UU.) ir a la guerra con Irak”. Así, el primer paso de la manipulación consiste, según los autores, en establecer el vínculo entre los ataques terroristas e Irak $(\mathrm{C}=$ el contexto del 11 de septiembre); el segundo consiste en bloquear el acceso a un contexto más amplio C', en el que Irak no está involucrado en los ataques a las Torres Gemelas.

Ahora bien, desde el punto de vista presentado en este trabajo, planteado así no se trataría de un caso de manipulación. Lo que ocurre aquí es simplemente que la razón que se ofrece (Hussein compró uranio de África) para la conclusión (Debemos declarar la guerra a Irak) es falsa o, más aún, es una mentira, pues como Maillat y Oswald (2009) señalan, la inteligencia norteamericana estaba al tanto de la falsedad de dichos datos. Por otra parte, el objetivo del agente manipulador aparece explicitado en la frase (Debemos declarar la guerra a Irak) que le es ofrecida al destinatario para ser interpretada a la luz de los supuestos contextuales parcializados o falsos. En este sentido, el que Hussein haya comprado uranio es una razón para la respuesta $\mathrm{R}_{1}$ (Debe declarársele la guerra a Irak) relativa a la cuestión $\mathrm{Q}_{1}$ ( Se le debe declarar la guerra a Irak?) que es explícitamente avanzada por el enunciador. Lo que encontramos aquí es simplemente una mentira o, en todo caso, si fuera cierta la información y Hussein hubiera comprado el uranio, se trataría de una argumentación en la que, a lo sumo, habría una utilización no atinente de los datos (pues no necesariamente puede inferirse su participación en los atentados del 11 de septiembre de la compra del uranio por parte de Irak).

En cambio, sí podríamos decir que se trataría de manipulación si consideramos que el objetivo ulterior de Bush y de EE.UU. no era simplemente declarar la guerra a Irak para desactivar el peligro terrorista que dicha nación representara (y exportar los valores democráticos), sino para controlar la producción y distribución del petróleo iraquí. En este caso, se presentaría de manera explícita R1 (Debe declarársele la guerra a Irak), relativa a Q1, y que sustituye a Q2 (¿Cómo puede EE.UU. controlar la producción y distribución del petróleo de Irak?). La relación entre R1 y R2 es de consecución: R1 es un paso en la dirección de conseguir R2, pues un modo de adueñarse del petróleo iraquí consiste en hacerse primero con el control militar y político de su territorio. Pero R1 no es R2 (identidad) ni supone realizar parcialmente R2 (inclusión), dado 
que luego de invadir el país otras circunstancias pueden impedir la explotación de los pozos petroleros por parte de EE.UU. (invadir el país no significa aún haber extraído su petróleo ni haberse beneficiado del mismo). En el caso de que el objetivo ulterior del agente manipulador sea apropiarse del petróleo (y no solamente hacer la guerra) existiría manipulación tanto si la información acerca de la compra de uranio fuera verdadera como si fuera falsa y, en ambos casos, ya sea que dicho agente fuera consciente o no de la falsedad de los datos, es decir, tanto si dijera la verdad como si mintiera.

\section{Conclusiones}

Hemos indicado que existe una imposibilidad de dar cuenta de la manipulación dentro de los límites de las teorías pragmáticas de los actos de habla (particularmente en la de Searle (2001), pero incluso en la de Grice, 2011): la noción de manipulación aquí presentada es perfectamente coherente con el hecho de que se pueda manipular sin violar máximas conversacionales (consideradas exclusivamente en términos de $\mathrm{Q}_{1}$ ), o bien, sin incumplir las condiciones de sinceridad (siendo quizás un problema de mayor envergadura y a investigar, el caso del acto de prometer), así como con el hecho de que no se pueda explicitar su fuerza ilocucionaria.

Del mismo modo, hemos indicado que la intención manipulativa no tiene, en principio, un estatus comunicativo, ya que la manipulación supone poner en relación dos cuestiones distintas de las que una sola se plantea explícitamente. Y la conexión entre las dos cuestiones es de carácter factual, es decir, dicha conexión no tiene nada que ver con las reglas constitutivas del lenguaje, sino que pertenece a la esfera de los hechos brutos o, en todo caso, al arreglo de medios afines al interior de la estrategia del agente manipulador. Señalamos, por otro lado, que la reputación de un hablante (en tanto conocido como manipulador), puede ser parte del contexto comunicativo e informativo a partir del que se realizan las movidas materiales (de contenido) de los turnos de habla en una conversación.

¿En qué consiste el proceso manipulativo en el contexto conversacional? Como vimos, no puede tratarse de la intención ilocucionaria, la cual tiene que ver con el acto de habla realizado por un enunciador, y que se resuelve en la fuerza ilocucionaria que dicho hablante espera que el destinatario reconozca a partir de su enunciado. No es, asimismo, asimilable sin 
más al acto perlocucionario, entendido como el efecto en las creencias o acciones del destinatario producido por el acto ilocucionario. Por último, tampoco tiene que ver con la intención manipulativa entendida solamente como las consecuencias prácticas que constituyen el objetivo ulterior del agente manipulador (apropiarse del petróleo de Medio Oriente en nuestro último ejemplo). En cambio, sostenemos que el sentido de la interacción manipulativa de un enunciado $E$, consiste en el vínculo fáctico que puede establecerse entre la cuestión $\mathrm{Q}_{1}$ que $E$ plantea (de manera explícita o implícita) y la cuestión no declarada $\mathrm{Q}_{2}$.

Podría pensarse que del mismo modo que es posible abordar el rol del influenciador de un modo teórico relativo a las estrategias conversacionales que en cada caso puede emplear, igualmente puede analizarse la manipulación en términos interactivos. Sin embargo, existe una diferencia crucial, que es que justamente la manipulación solo deja como huella el tipo de vínculo que podría establecerse entre $\mathrm{Q}_{1}$ y $\mathrm{Q}_{2}$ en un contexto determinado. Este vínculo, por no ser de tipo convencional, no puede ser establecido a menos que los acontecimientos hayan revelado dicha conexión, o bien, que el agente manipulador confesara su intención o que la evidencia circunstancial fuera abrumadora.

La manipulación puede ser, en ciertos casos, el reverso de la falacia ad hominem circunstancial: quien comete esta falacia puede estar acusando a alguien de manipulación. Consideremos la siguiente frase: "A me dijo que el auto X es el mejor del mercado, pero luego supe que él tiene acciones en la compañía que lo fabrica”. ¿Qué quiere decir esto? En primer lugar, parece indicar que la manipulación debe ser probada fehacientemente, porque la acusación de manipulador constituye una falacia en ciertos casos; es decir, no solamente hay que demostrar que efectivamente tenía acciones, sino que en realidad sabía que el auto no era bueno y que lo que dijo fue expresado solamente por los beneficios que una venta más acarrearía a la empresa. En segundo lugar, sugiere que la detección de la manipulación puede involucrar la puesta en relación de los contenidos del enunciado con datos de un orden diferente de cosas (o la puesta en relación de dos cuestiones), que a priori no se habría buscado: el lugar en donde trabaja el enunciador no tiene relación alguna con las cualidades del automóvil sobre las que se está discutiendo.

Desde el punto de vista del destinatario, como señalan Maillat y Oswald (2009), la manipulación supone la imposibilidad de acceder a información crítica que le permitiría interpretar el enunciado de manera 
satisfactoria. Desde el punto de vista de la interacción conversacional como producto, la manipulación supone una sustitución estratégica de Q2 por Q1, la cual es capaz de producir una respuesta R1 vinculada en la práctica - ya sea por equivalencia, inclusión/intersección, consecución-con R2, que representa el objetivo ulterior del enunciador. Conviene hablar de estrategia de manipulación y no ya de intención manipulativa, puesto que esta última idea remite fundamentalmente a la finalidad del engaño, mientras que al emplear los términos de estrategia de manipulación, se pone el foco en el cálculo que supone la conexión entre la cuestión explícita Q1 y la que no se explicita Q2 en el marco de intercambios conversacionales.

\section{Referencias bibliográficas}

Austin, J. (1998). Cómo hacer cosas con palabras. Barcelona: Paidós.

Bremond, C. (1970). "Le rôle d'influenceur". Communications 16(1), 60-69. https://doi.org/10.3406/comm.1970.1229

Fillmore, C. (1982). Frame semantics. En Linguistic Society of Korea (Ed.), Linguistics in the morning calm (pp. 111-138). Seoul: Hanshin.

Grice, H. P. (2011). Studies in the way of words. Cambridge, MA: Harvard University Press.

Lakoff, G. (1993). The contemporary theory of metaphor. En A. Ortony (Ed.), Metaphor and thought (pp. 202-251). Cambridge, MA: Cambridge University Press.

Lakoff, G. (1994). What is conceptual system? En W. Overton \& D. Palermo (Eds.), The nature and ontogenesis of meaning (pp. 41-90). Hillsdale, NJ: Lawrence Erlbaum.

Lakoff, G. \& Johnson, M. (1980). Metaphors we live by. Chicago: University of Chicago Press.

Langacker, R. (1987). Foundations of cognitive grammar (Vol. 1). California: Stanford University Press.

Landowski, E. (2005). Tres regímenes de sentido e interacción. Tópicos del Seminario, 14, 137-179. Recuperado de http://www.redalyc. org/articulo.oa?id=59401408

Macagno, F. (2014). Manipulating emotions. Value-based reasoning and emotive language. Argumentation and advocacy, 51(2), 103-122. https://doi.org/10.1080/00028533.2014.11821842 
Maillat, D. \& Oswald, S. (2009). Defining manipulative discourse: The pragmatics of cognitive illusions. International Review of Pragmatics, 1(2), 348-370. https://doi.org/10.1163/18773090 9X12535267111651

Meyer, M. (2013). Principia rhetorica. Una teoría general de la argumentación. Buenos Aires: Amorrortu Editores.

Plantin, C. (1993). Lieux communs dans l'interaction argumentative. En C. Plantin (Ed.), Lieux communs, topoi, stéréotypes, clichés (pp. 480-496). Paris: Kimé.

Real Academia Española, RAE. (2014). Diccionario de la lengua española (23 ed.). Madrid: Espasa Calpe.

Santibáñez, C. (2017). Strategically wrong: On the relationship between generalized deception and persuasive behavior. Journal of Pragmatics, 114, 16-31. https://doi.org/10.1016/j. pragma.2017.04.001

Searle, J. (2001). Actos de habla. Madrid: Cátedra.

Searle, J. (2002). How performatives work. En J. Searle (Ed.), Consciousness and language (pp. 156-179). New York: Cambridge University Press.

Sperber, D. \& Wilson, D. (1995). Relevance. Communication and cognition. Oxford: Blackwell.

van Dijk, T. (2006). Discurso y manipulación: Discusión teórica y algunas aplicaciones. Revista Signos, 39(60), 49-74. https://doi. org/10.4067/s0718-09342006000100003

Webster's Encyclopedic. (1996). Unabridged dictionary of the English language. New York: Random House. 